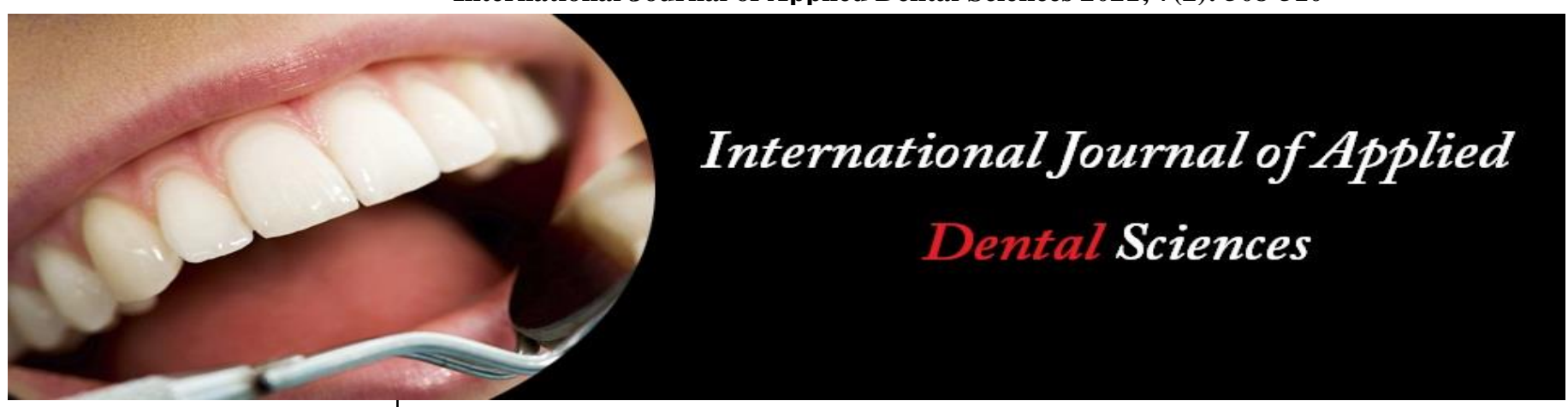

ISSN Print: 2394-7489

ISSN Online: 2394-7497

IJADS 2021; 7(2): 508-510

(C) 2021 IJADS

www.oraljournal.com

Received: 12-02-2021

Accepted: 15-03-2021

Dr. Srivani Kambhampati

MDS, Assistant Professor (Senior

Lecturere), Department of

Prosthodontics, Sri Sai College of

Dental Surgery, Vikarabad,

Telangana, India

Dr. Sushma Kavuri

MDS, Department of

Prosthodontics, Private Dental

Practitioner, Vijayawada,

Andhra Pradesh, India

Dr. Chandra Deepika Chundury MDS, Department of

Prosthodontics, Private Dental

Practitioner, Vijayawada,

Andhra Pradesh, India

Dr. Pavani Pothabathula

MDS, Department of

Prosthodontics, Private Dental

Practitioner, Hyderabad,

Telangana, India

\section{Evaluation of Shear bond strength values of PEEK to dentin using different surface treatments}

\author{
Dr. Srivani Kambhampati, Dr. Sushma Kavuri, Dr. Chandra Deepika \\ Chundury and Dr. Pavani Pothabathula
}

DOI: $\underline{\text { https://doi.org/10.22271/oral.2021.v7.i2h.1254 }}$

Abstract

Aim: To compare the shear bond strength values of PEEK dentin using different surface treatment by sandblasting and acid etching techniques.

Methodology: Forty removed human molar teeth (maxillary/mandibular) were used in this investigation. Extracted teeth were cleaned with an ultrasonic scaler (EMS, Switzerland) and kept in a $10 \%$ formalin solution to eliminate surface debris. An acrylic resin block of dimensions $10 \mathrm{~mm}$ x10mm was fabricated using autopolymerising acrylic resin (DPI-RR Cold Cure, India) and the teeth were embedded in the center of block upto complete submergence of the root. Two groups were made (group A and group B), each comprising of 20 blocks and colour coding was done to distinguish the groups. Group A comprised of clear acrylic resin blocks in which the PEEK surfaces were modified by acid etching and group B comprised of pink acrylic resin blocks in which PEEK surface was modified by sandblasting.

Results: Mean \pm SD of shear bond strength recorded for group A and B was 1.94 and $2.40 \mathrm{Mpa}$ respectively with statistically significant difference as $P<0.05$.

Conclusion: It can therefore be concluded that surface modification with sandblasting is favored over acid etching to achieve good bonding between PEEK and dentin.

Keywords: PEEK, dentin, acid etching, sandblasting

\section{Introduction}

Polyetheretherketone (PEEK) is semi crystalline high-performance polymer synthesized from aromatic dihalides and biphenolate salts via nucleophilic substitution ${ }^{[1]}$. PEEK material can be sterilized by radiation and heat without structural damage thus making it an attractive biomaterial for medical use ${ }^{[2]}$.

Despite having great mechanical qualities for dental prosthesis, PEEK does not meet the cosmetic requirements. According to a recent study, pre-treating PEEK surfaces with sulfuric acid or mechanical abrasion with aluminum oxide greatly boosted bond strength ${ }^{[3]}$.

Previous research examined resin cement adherence to PEEK with and without surface preparation to lute the repair to dentin. Surface treatments such as sandblasting with alumina and silica coating (Rocatec) were evaluated in these investigations ${ }^{[4]}$. Other experiments looked at more experimental surface treatments for dental PEEK, such as 98 percent sulfuric acid attack at different times of exposure and inert plasma therapy with cold active gas. PEEK surface treatments were shown to be suitable for adhesion to resin cement to further lute the dentin restoration in these investigations ${ }^{[3]}$.

Due to limited data availability, this study was undertaken to compare shear bond strength values of PEEK dentin using different surface treatment by sandblasting and acid etching techniques.

\section{Material and Method}

Using two separate procedures, this in-vitro investigation was carried out to assess the binding strength of surface modified PEEK (Polyether-Ether-Ketone) to dentin. A total of forty extracted human molar teeth (maxillary/mandibular) were used in this investigation. Extracted teeth were cleaned with an ultrasonic scaler (EMS, Switzerland) and kept in a $10 \%$ formalin
Dr. Srivani Kambhampati

MDS, Assistant Professor (Senior

Lecturere), Department of

Prosthodontics, Sri Sai College of

Dental Surgery, Vikarabad,

Telangana, India 
solution to eliminate surface debris. The teeth were inserted in the core of the acrylic resin block up to total submergence of the root (DPI-RR Cold Cure, India) using auto-polymerising acrylic resin (DPI-RR Cold Cure, India). Two groups (group A and group B) were created, each with 20 blocks, and color coding was used to separate the two groups. Group A consisted of clear acrylic resin blocks with acid etching on the PEEK surfaces, while group B consisted of pink acrylic resin blocks with sandblasting on the PEEK surfaces.

\section{Inclusion criteria}

Intact maxillary/mandibular molar teeth.

\section{Exclusion criteria}

Decayed/mutilated teeth, Restored teeth, Root canal treated tooth, Teeth with wasting disease such as attrition, abrasion, erosion and tooth with periapical pathology.

The teeth were embedded up to their roots in the center of auto-polymerising acrylic resin block measuring $10 \mathrm{mmx} 10 \mathrm{~mm}$. These acrylic blocks were color coded and randomly divided into two groups (20 blocks per group), according to surface treatment. The selected teeth were embedded along their long axis with the cemento-enamel junction positioned $1 \mathrm{~mm}$ above the top of the custom-made mounting base. The mounting base was fabricated using autopolymerising acrylic resin using a custom-made mounting template which had a slot measuring $10 \mathrm{~mm} \times 10 \mathrm{~mm}$, into which the acrylic resin was poured in the center and selected tooth was placed into the base before the complete curing of acrylic resin, the acrylic block with mounted tooth was removed from custom made mounting base after complete setting of acrylic resin. Vertical alignment of the teeth was verified using a milling surveyor. The teeth with mounting block were kept in artificial saliva at room temperature after each procedure performed on the blocks.

Forty PEEK disks with dimensions of $3 \mathrm{~mm}$ diameter and $3 \mathrm{~mm}$ thickness were prepared from breCAM. BioHPP blank (Bredent group) using CAD/CAM technology. A file for PEEK disk was designed with 3D printing software and this file was transferred to CAD software and after nesting of disk in PEEK blank, the disk was milled using milling machine (Yenadent D 43 Turkey). The PEEK disk surfaces of group A and group B were then subjected to treatment with acid etching and sandblasting respectively as under:

(A) 20 samples of group A were treated with acid-etching using sulfuric acid (98\%) for $1 \mathrm{~min}$ followed by careful rinsing with de-ionized water for $1 \mathrm{~min}$.

(B) 20 samples of group B were treated using sandblasting with alumina for $15 \mathrm{~s}$ at a pressure of 2.8 bar. A distance of 1 $\mathrm{cm}$ was maintained between the nozzle and the surface.

\section{Shear bond strength test (SBS)}

After luting, samples were tested to evaluate if there were differences in SBS between PEEK and dentin depending on the use of surface treatment. Shear bond strength of specimens was tested by Universal Testing Machine (Model H $25 \mathrm{KS}$, Birmingham, United Kingdom). The device for applying shear force was mounted in the upper jaw and the prepared specimens were mounted in the lower jaw of the Universal Testing Machine. Specimens were tested in the parallel shear test, under a constant load of $5000 \mathrm{~N}$ with a crosshead speed of $0.5 \mathrm{~mm} / \mathrm{min}$. The force was applied at the interface of the tooth surface and the bonded disc. The machine recorded the amount of force required for debonding of the disks in Newton (N). The shear bond strength was recorded by dividing the load by the bonding surface area (7.07 sqmm) and the result of SBS was measured in MPa.

\section{Statistical analysis}

The means and standard deviations of the measurements per group were used for statistical analysis (SPSS 24.00 for windows; SPSS inc, Chicago, USA). For each assessment point, data were statistically analysed using $t$ test and the level of significance was set at $p<0.05$.

\section{Results}

In the current study, mean \pm SD of shear bond strength recorded for group A and B was $1.94 \pm 0.84$ and $2.40 \pm 0.82$ Mpa respectively with statistically significant difference as $P<0.05$ (table 1).

Table 1: Shear bond strength (Mpa) of Group A (Acid etching) and Group B (Sandblasting)

\begin{tabular}{|c|c|c|c|c|c|}
\hline $\begin{array}{c}\text { Shear bind } \\
\text { strength }\end{array}$ & Mean & SD & Minimum & Maximum & Median \\
\hline Group A & 1.94 & 0.84 & 0.36 & 3.44 & 1.66 \\
\hline Group B & 2.40 & 0.82 & 1.15 & 4.31 & 2.14 \\
\hline \multicolumn{3}{|c|}{ t test } & \multicolumn{3}{c|}{2.29} \\
\hline \multicolumn{3}{|c|}{ p value } & & \\
\hline
\end{tabular}

*: Statistically significant

\section{Discussion}

The mean \pm SD shear bond strength obtained for group A in this investigation was $1.94 \pm 0.84 \mathrm{Mpa}$. PEEK treated by 98 percent concentrated sulphuric acid, have a very porous and sticky permeable surface area in previous research ${ }^{[5]}$. Bond strength increased as a result of this. Despite this, because of the presence of hydrochloric and nitric acid, the surface structure of PEEK cannot be altered. PEEK sulfuric acid tag development was not seen by Schimidlin et colleagues ${ }^{[6]}$. Increasing pores result from increased sulfuric acid exposure duration. The longer the time of exposure, the worse the PEEK layer, the more coherent it may result. A higher percentage of the PEEK cohesive failures were found when exposed to 90,120 or 300 s as correlated in a study conducted by Sproesser et al. ${ }^{[7]}$.

The mean \pm SD shear bond strength obtained for group B in this study was $2.40 \pm 0.82 \mathrm{Mpa}$. Sandstorms can change the shape of the PEEK layer, improving micro-mechanical interlocking and, in turn, the strutting cement's ability to penetrate the composite material. It is clear that group B's shear bond strength $(2.40 \mathrm{Mpa})$ was higher than group A's $(1.90 \mathrm{Mpa})$, with a statistically significant difference of $P<0.05$. The findings were comparable to those of Stawarczyk et al. study ${ }^{[8]}$. In their investigation, Rocha RFV et al. ${ }^{[3]}$ reported comparable results.

It's important to remember that sulfuric acid at such a high concentration (98\%) is hazardous, since it can inflict major damage upon contact, making it impracticable to use on the chair or in the lab. Industrial milling and processing of PEEK with 98 percent sulfuric acid might be an appropriate approach. However, before bonding, pretreatment surface contamination in the dental laboratory and office must be prevented or eradicated.

\section{Conclusion}

As a result, it may be stated that sandblasting is preferable to acid etching for achieving excellent bonding between PEEK and dentin. On the basis of this in vitro investigation, only the influence of various surface modification techniques on bond 
strength can be determined, which can offer indicator of the clinical phase. For human patients, for in vivo trials are advised to compare the results obtained with in vitro studies in order to investigate the long-term success of PEEK under actual conditions.

\section{References}

1. Yang J, Gibson HW. A polyketone synthesis involving nucleophilic substitution via carbanions derived from bis( $\alpha$-aminonitrile)s. 5.1-4 A new, well-controlled route to "long" bisphenol and activated aromatic dihalide monomers. Macromolecules 1999;32(26):8740-6.

2. Green S, Schlegel J. A polyaryletherketone biomaterial for use in medical implant applications. Polym for the Med Ind Proc, Brussels 2001, 14-5.

3. Rocha RFV, Anami LC, Campos TMB, de Melo RM, e Souza RO de A, Bottino MA. Bonding of the polymer polyetheretherketone (PEEK) to human dentin: Effect of surface treatments. Braz Dent J 2016;27(6):693-9.

4. Fuhrmann G, Steiner M, Freitag-Wolf S, Kern M. Resin bonding to three types of polyaryletherketones (PAEKs) Durability and influence of surface conditioning. Dent Mater 2014;30(3):357-63.

5. Zhou L, Qian Y, Zhu Y, Liu H, Gan K, Guo J. The effect of different surface treatments on the bond strength of PEEK composite materials (DEMA-D-13-00481). Dent Mater 2014;30(8):1-7.

6. Schmidlin PR, Stawarczyk B, Wieland M, Attin T, Hämmerle $\mathrm{CH}$, Fischer J. Effect of different surface pretreatments and luting materials on shear bond strength to PEEK. Dental materials 2010;26(6):553-9.

7. Sproesser O, Uhrenbacher J, Gernet W, Stawarczyk B, Schmidlin PR, Roos M. Effect of sulfuric acid etching of polyetheretherketone on the shear bond strength to resin cements. J Adhes Dent 2014;16(5):465-72.

8. Stawarczyk B, Beuer F, Wimmer $\mathrm{T}$ et al. Polyetheretherketone - A suitable material for fixed dental prostheses? J Biomed Mater Res - Part B Appl Biomater 2013;101(7):1209-16. 